\title{
Industrial Applications
}

\section{I.J. Spalding, Culham}

\author{
(U.K. AEA Laser Applications Group)
}

Lasers now have practical uses which span a range of powers extending from microwatts to 100 terawatts. A wide variety of useful and reliable ultra-violet, visible and infrared lasers are already available commercially whilst the primary wavelengths available from laboratory devices straddle the electromagnetic spectrum from $\lambda \sim 18 \mathrm{~nm}$ to more than $1 \mathrm{~mm}$ (though not yet continuously). The laser field then, is very broad - and the range of industrial applications correspondingly diverse. Some generalisations are, nevertheless, possible.

The directionality of the $\mathrm{He}-\mathrm{Ne}$ laser beam is readily exploited for alignment and surveying (CERN accelerators, San Francisco Bay dredging, measuring deck movements on super-tankers etc.). If the output is also monochromatic (as it is in most lasers) the coherence of the light source lends itself to extremely accurate measurements of position: for example, Tozer of the UK Central Electricity Generating Board has used holographic techniques to make measurements of position in remote radioactive environments with a resolution of $10 \mu \mathrm{m}$ and a depth of field exceeding $1 \mathrm{~m}$. Similarly, reflection measurements can be employed for the high-speed monitoring of surface quality, as in the paper scanner marketed by Ferranti Ltd.

The frequency stability (quite typically 1 part in $10^{8}$ to $10^{10}$ ) can be applied to velocity measurements, as in the scanning laser doppler anemometers (E.O. Schulz-Dubois, EN, 11 (1980) 10, 9), which measure, e.g. atmospheric turbulence on runways at air fields. Wide bandwidth (and therefore short pulse) diode and other lasers are used increasingly for communications and datatransmission, e.g. the $140 \mathrm{M} \mathrm{bit/s}$ optical telephone link, and fully tunable lasers for pollution-monitoring and similar transmission and scattering measurements. An interesting wavelength-sensitive application is the inspection of faults in the polyethylene insulation on cables; Cheo, at the United Technologies Research Centre, has discussed the performance of a prototype system which uses a $\mathrm{CH}_{3} \mathrm{OH}$ laser at $119 \mu \mathrm{m}$ for this purpose.

To avoid too many generalities let us consider what is perhaps the longest established laser application, the use of solid-state and gas-lasers for the heating (and thus materials-processing) of engineering or other workpieces. For such work the precise choice of wavelength is normally of less importance than the total power and focal intensity applied to the target.
(An interesting exception is the laserannealing of semiconductor materials, M. Bertolotti, EN, 10 (1979) 12, 9).

\section{Industrial Materials Processing}

A well-focused laser beam can provide a uniquely high power density, very precisely localized in space. In this respect it has similar characteristics to an electron beam, over which it holds the following advantages:

It is insensitive to electric and magnetic fields, can be used on re-entrant surfaces, does not require a vacuum, and is therefore more readily transmitted and manipulated over long distances $(30-50 \mathrm{~m})$ in difficult (e.g. radioactive) environments.

In consequence, lasers offer considerable technical potential for well-controlled, low distortion, high-speed, single-pass cutting and welding of metals, and some plastics. Alternatively, the beam can be manipulated to produce a broader heating pattern at the workpiece, suitable for a range of surface-treating processes.

Many non-metals can be cut effectively and economically at mean laser powers of between 5 and $500 \mathrm{~W}$ using $\mathrm{Nd}-\mathrm{YAG}$ or $\mathrm{CO}_{2}$ lasers. One such application is the cutting of quartz-ware by a continuous $400 \mathrm{~W} \mathrm{CO}$ laser; advantages of the process are the "flame-polished" edge (which reduces subsequent handling damage arising from crack propagation), and the reduction in the number of operations associated with diamond-wheel cutting. Many other interesting applications exist in the packaging, printing, plastics and related industries. Two well-publicized examples are the automatic cutting of die-boards (which hold the cutting and bending blades used to form cigarette and other cartons), and the production of rotogravure printing cylinders by on-line "dry" techniques. Here, the laser is used as a very advanced machine tool. Another mass-production application has recently been introduced by the Bank of England, for its new $£ 50$ note. Within the note is a Security Thread, contoured to a pattern that is cut at high speed by a focused laser beam, steered by a microprocessor-controlled optical system.

Machining and welding metals thicker than a few $\mathrm{mm}$ normally require higher powers (because of heat conduction effects) and here the $\mathrm{CO}_{2}$ laser has the dual advantages of scalability and relatively high $(\sim 10 \%)$ efficiency. The Table summarizes typical cutting and welding speeds attainable within the power range $P=400 \mathrm{~W}$

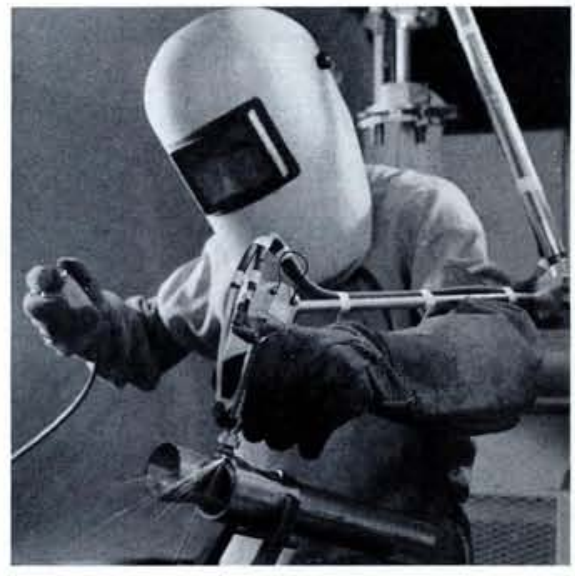

Fig. 1 - $400 \mathrm{~W}$ flexible laser beam guide $112 \mathrm{~mm}$ bore).

to $5 \mathrm{~kW}\left(P\right.$ is the $\mathrm{CO}_{2}$ laser power actually focused on to the workpiece). Cutting speed can be significantly enhanced by using high pressure jets of oxygen-enriched or normal air (rather than an inert gas) although the cut quality deteriorates in consequence. In welding, the cooling surface, is normally shrouded by an inert gas and a localized helium jet is also used to provide adequate control of the laserplasma produced.

Cutting and welding performance depends on mode quality as well as the power delivered to the workpiece, the latter depending on the total number of optical components $(N)$ in the beam delivery line. The power $P_{0}$ of the laser required is: $P_{0}=P T^{-N}$, where $T$ is the mean transmission of an optical element. The transmission loss may approach $25 \%$ for a reasonably complex device, such as the $400 \mathrm{~W}$ flexible beam guide illustrated in Fig. 1. This device is used to couple the laser beam to three-dimensional work surfaces, or to facilitate alignment in difficult environments. Fig. 2 is a photograph of welds made in $9 \mathrm{~mm}$ thick stainless steel using

Illustrative Cutting and Welding Data

\begin{tabular}{lccc}
\hline Power on Work $(P)$ & 350 W & 4.6 kW \\
\hline & & $\begin{array}{c}\text { Typical Work Speed } \\
\text { (mm/s) }\end{array}$ \\
\hline Material & Thickness $(\mathrm{mm})$ & \multicolumn{2}{c}{ Cutting } \\
\hline Mild Steel & 2.45 & - & $100^{\mathrm{a}}$ \\
PE 16 & 2.62 & $3.8^{\mathrm{b}}$ & - \\
PE 16 & 2.62 & $8.5^{\mathrm{c}}$ & - \\
Stainless & 5.00 & $1.9^{\mathrm{b}}$ & - \\
Mild Steel & 6.00 & - & $30^{\mathrm{d}}$ \\
\hline & & - & Welding \\
\hline Stainless & 5 & - & $15^{\mathrm{d}}$ \\
Stainless & 8 & - & $10^{\mathrm{d}}$ \\
Stainless & 9 & - & $8^{\mathrm{d}}$ \\
\hline
\end{tabular}

(a) Air jet.

(b) $\mathrm{O}_{2}$ jet $-17 \mathrm{I} / \mathrm{atm} \mathrm{min}$

(c) $\mathrm{O}_{2}$ jet $-27 \mathrm{I} / \mathrm{atm} \mathrm{min}$

(d) He jet - giving high quality oxide free cut or weld 


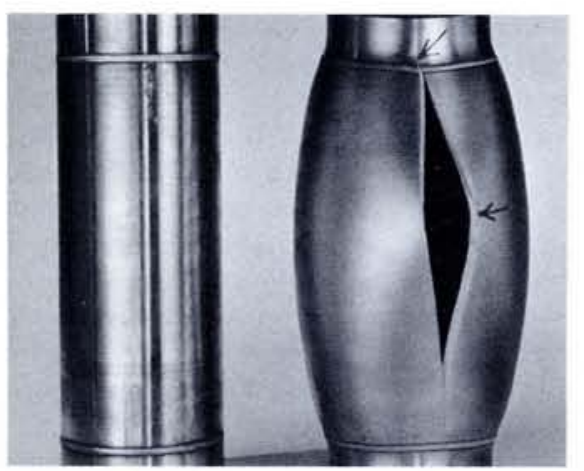

Fig. 2 - Pressure vessel test on CL5 laser-weld in $9 \mathrm{~mm}$ stainless steel. The crack initiates at a stress raiser and runs cleanly across the weld.

such equipment, before and after being subjected to a standard pressure-vessel test. Allowing for beam-delivery losses in typical "production-line" systems, reliable welding at thickness greater than about $12 \mathrm{~mm}$ demands powers in the $6-10 \mathrm{~kW}$ range, or greater.

Two interesting sub-kilowatt laser cutting systems have been in use at the fast reactor centre of Dounreay. The first is at the post-irradiation examination cell for "topping and tailing" irradiated nuclear fuel subassemblies before they are sent in a transit can to the reprocessing building The second is at the dis-assembly cave to open the transit can, and section the fuel wrapper.

As noted previously, the ability to scan high-intensity, carefully-localized heat patterns over metallic surfaces provides the opportunity for unusual case-hardening or other surface treatments, using basically the same laser equipment. Here, the potential technical and economic advantages over more conventional surface processes include faster production cycles, less distortion resulting in fewer rejects, and the elimination of post heat-treatment materials-processing. Laser hardened parts may be placed directly on the final assembly line, without costly grinding, honing, and straightening operations. In order of in creasing power density, process complexity, and decreasing state of development, four distinct processes may be identified: 1. Martensitic transformation-hardening.

2. Surface alloying and cladding.

3. Creation of rapidly-quenched surface layers (laser and layer glazing).

4. Shock hardening.

\section{Conclusion}

Only some of the many exciting and in novative areas of application for lasers in industry have been outlined, but as constraints such as capital costs, running costs and system reliability become less restrictive, the areas continue to grow. In massproduction applications, and in markets which are sufficiently large for the laser system itself to be mass produced, such constraints are being considerably reduced all the time.

\title{
Optical Storage on Discs
}

\section{G. Bouwhuis and J.J.M. Braat, Eindhoven}

\author{
(Philips' Research Laboratories)
}

When storing information by optical means ${ }^{1}$ ), enormous densities of up to $10^{8}$ bits $/ \mathrm{cm}^{2}$ are nominally possible and, with the aid of a spinning disc, high bit rates and random access are readily feasible. To record or scan the information track, it is clear that an adequate power has to be delivered to the scanning spot. For reading the data, the signal to noise ratio should be at least $20 \mathrm{~dB}$, and in any case, the shot noise (photon noise) has to be well below this value. This means that for a data rate of $10 \mathrm{Mb} / \mathrm{s}$, the power transmitted by the source through the optical system to the information spot has to be above $0.3 \mu \mathrm{W}$. At the same time, the size of the spot, limited by diffraction, implies a radiance that is certainly larger than $30 \mathrm{~W} / \mathrm{cm}^{2}$ ster. This can not be attained with conventional sources owing to the short life-time or, with gas discharge lamps for example, the source noise.

For video discs, a signal to noise ratio of at least $35 \mathrm{~dB}$ of the photosignal is needed, demanding a radiance more than an order of magnitude larger. In these circumstances, a laser is the only possible source of itlumination. Moreover, the power and beam quality that can be obtained from a laser, opens the possibility of recording by burning holes in a thin layer. Holes of $0.8 \mu \mathrm{m}$ diameter can be formed in tellurium layers with an incident energy of about $1 \mathrm{~nJ}{ }^{2}$ ), demanding a peak power from the laser source of at least $50 \mathrm{~mW}$.

Early optical data recorders made use of gas lasers like the $1 \mathrm{~W}$ argon or krypton ion laser with, for modulation, an electrooptical or acousto-optical device. Recent progress however, in the development of the semiconductor $\mathrm{AlGaAs}$ laser has led to peak powers of up to about $100 \mathrm{~mW}$ being available which is amply sufficient for hole burning. A very attractive property of the semiconductor laser is that its output can be directly modulated with the drive current. Data rates of up to $30 \mathrm{Mb} / \mathrm{s}$ have been achieved ${ }^{3}$ ).

In order to attain the high information density, the scanning spot has to be as small as possible, typically of the order of $1 \mu \mathrm{m}$ diameter. Such sizes can be obtained only with the aid of a fairly high numerical aperture of the spot-forming optics, which should produce virtually diffraction limited quality. The small size of the spot also requires the elimination of transverse modes in the beam. The longitudinal modes of AIGaAs lasers often show non-circular symmetry both in amplitude and phase distribution, but as the mode is basically diffraction limited, it can be fully adapted to the optics at the cost of a more complicated light path. In practice, the phase asymmetry is corrected by a weak cylindrical lens and although optical systems correcting the amplitude asymmetry exist, a simple optical system that collects only the central, uniform, part of the beam, is generally used.

As the spot diameter is proportional to wavelength, a small wavelength of the laser light is advantageous, and in this respect the $\mathrm{He}-\mathrm{Ne}$ laser with a wavelength $20 \%$ shorter would be preferred to the present AlGaAs laser. Translated to the playing time of a video disc, the shorter wavelength implies a $40 \%$ gain, which is significant, since increasing the playing time by increasing the value of the numerical aperture, leads to tighter tolerances being imposed on the optical system and the disc parameters.

The effective spectral width of the source is important for the design of the spot-forming lens. Both types of laser are sufficiently monochromatic for a oneelement aspherical lens to be used. However, a very small spectral width (a long coherence length) gives rise to unwanted interference effects due to light reflection at lens surfaces or diffusion by dust and scratches. Moreover, coherent feedback of radiation to the source may modulate the output of the laser. When using a $\mathrm{He}-\mathrm{Ne}$ laser this is a nuisance whereas feedback to the AIGaAs laser can be exploited advantageously. A spot imaged on a reflecting information layer is inevitably re-imaged onto the source itself, and if no special means (e.g. polarized optics) are taken to avoid feedback, the output of the AIGaAs laser will vary according to the data in the track. The output modulation can thus be measured with a photodetector on the rear side of the laser, or directly in the driving circuitry: the source becomes then also the detector.

From the above, it can be concluded that the development now of high power AlGaAs lasers with long lifetime at room temperature has made optical recording a practical and powerful medium.

\section{REFERENCES}

1. Seven articles in Applied Optics, 17 (1968) 1993-2042.

2. Bulthuis K. et al., IEEE Spectrum, (1979) August, p. 26.

3. Bartolini R.A. et al., IEEE J. Quantum Electr., 17 (1981) 69 . 